Résumés des conférences et travaux

\title{
Histoire et philologie du Japon ancien et médiéval
}

\author{
Charlotte von Verschuer
}

\section{OpenEdition \\ Journals}

Édition électronique

URL : https://journals.openedition.org/ashp/1376

DOI : 10.4000/ashp.1376

ISSN : 1969-6310

Éditeur

Publications de l'École Pratique des Hautes Études

Édition imprimée

Date de publication : 1 octobre 2012

Pagination : 334-340

ISSN : 0766-0677

Référence électronique

Charlotte von Verschuer, « Histoire et philologie du Japon ancien et médiéval », Annuaire de l'École pratique des hautes études (EPHE), Section des sciences historiques et philologiques [En ligne], 143 | 2012, mis en ligne le 28 septembre 2012, consulté le 03 août 2021. URL : http://journals.openedition.org/ ashp/1376 ; DOI : https://doi.org/10.4000/ashp.1376 


\title{
HISTOIRE ET PHILOLOGIE DU JAPON ANCIEN ET MÉDIÉVAL
}

\author{
Directeur d'études : $\mathrm{M}^{\text {me }}$ Charlotte von Verschuer
}

Programme de l'année 2010-2011 : I. L'économie du sanctuaire d'Ise aux IXe-Xe siècles. - II. "Chronique des relations avec les pays voisins » (Zenrin kokuhôki, 1470), texte en kanbun (suite).

\section{L'économie du sanctuaire d'Ise aux IX'e-Xe siècles}

Les origines du sanctuaire d'Ise (département de Mie) sont mal connues. Selon la mythologie, les souverains du Yamato auraient fondé cet établissement au $\mathrm{I}^{\mathrm{er}}$ siècle de notre ère pour vénérer la déesse du soleil Amaterasu. Les « Chroniques du Japon » (Nihon shoki, 720 apr. J.-C.) mentionnent une exemption d'impôts de la province d'Ise que l'impératrice Jitô (r. 686-697) aurait ordonnée en 692, à la suite d'un oracle reçu de la déesse du soleil, et pour l'année 698, ce même texte note le transfert du « grand sanctuaire » dai jing $\hat{u}$, du district de Take vers celui de Watarai, toujours dans la province d'Ise. Le sanctuaire d'Ise est alors administré par la cour impériale installée à Fujiwara-kyô (694-710). Il se compose d'un sanctuaire intérieur naigûu - consacré à Amaterasu et ses deux assistants, le dieu Ameno-chikara no mikoto et la déesse du tissage Yorozuhata-toyoakitsu hime -, et d'un sanctuaire extérieur gek $\hat{u}$, siège du dieu Toyke ômikami, dieu de l'agriculture qui gère les repas quotidiens de la déesse Amaterasu. C'est à cette époque qu'une première structure fiscale voit le jour, à savoir l'établissement de deux « districts du sanctuaire » shingun, Take et Watarai, qui seront plus tard rejoints par les districts de Iino et de Iitaka. La majorité (ou la totalité?) des foyers de ces districts deviennent des «foyers concédés au sanctuaire » fuko ou «foyers du sanctuaire » shingo ou kanbe. Les impôts des « districts du sanctuaire » sont gérés par le gouverneur de la province d'Ise qui fait rapport au gouvernement central, c'est-àdire la cour impériale de Nara (710-784), et plus tard à celle de Heian (794-1192).

Après avoir clarifié ces premières données à partir des chroniques Nihon shoki, Shoku nihongi (797) et du code administratif ritsuryô publié en 701, nous avons consacré nos séances à la gestion économique du sanctuaire telle qu'elle apparait, entre autres, dans les « Rapports sur le protocole du Sanctuaire impérial d'Ise » Kôtai jingû gishikichô (ci-après abrégé Kjg) et du « Sanctuaire extérieur Toyuke » Toyuke

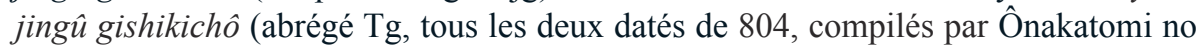
Masatsugu, coll. " Shintô taikei », Jingû hen 1), ainsi que dans le Livre 4 des « Règlements de l'ère Engi » Engishiki (927 apr. J.-C., Fujiwara no Tokihira et al., édition de Torao Toshiya, Shûeisha, vol. 1, 2005, ci-après abrégé ES), qui est consacré à la gestion du sanctuaire d'Ise. Les ressources du sanctuaire sont relativement connues grâce aux documents administratifs et législatifs, mais le budget annuel a été peu étudié, parce qu'il doit être reconstitué à partir de cas particuliers. On est amené à recourir aux estimations. De manière générale, les dépenses annuelles du sanctuaire comprenaient 
l'entretien des bâtiments, les équipements, le personnel, les rites annuels, les offrandes quotidiennes et la gestion courante. Nous avons essayé de collecter des données chiffrées sur le fonctionnement du sanctuaire.

Le sanctuaire d'Ise célébrait le rite des « Travaux agricoles au fil des mois » Tsukinami-sai au sixième et au douzième mois, et celui des « Prémices » Kanname, au neuvième mois (avant les « Prémices » de la cour impériale Niinamesai du onzième mois), appelés les « trois grands rites » san-sechi ou mi-ori, et plusieurs autres rites annuels. Pour les seuls «trois rites », on fabriquait chaque année au total 2349 objets comprenant du mobilier et plus d'un millier d'emballages tsutsumi pour le riz d'offrande, vraisemblablement faits de nattes de paille (Kjg, p. 123). Pour le rite du sixième mois, on préparait les denrées suivantes (Kjg, p. 150) : 38,4 koku (3064 litres ou à peu près 2 tonnes) de riz, un koku (80 litres) de sel, 20 jarres de saké d'offrande miki, 25 charges ni de denrées fraîches nie, 10 livres $(6,74 \mathrm{~kg})$ de ouate de fibre végétale $y \hat{u}, 1,3$ livres $(0,9 \mathrm{~kg})$ de fibre de chanvre $a s a, 40$ écheveaux de fil rouge et un lingot de fer. Ces quantités, qui concernaient le sanctuaire intérieur, étaient légèrement inférieures pour le sanctuaire extérieur, par exemple 37 koku de riz au lieu de 38,4 (Tg, p. 274). On peut estimer à 12 tonnes la quantité de riz utilisée par le sanctuaire d'Ise pour les « trois grands rites » de l'année, y compris le riz d'offrande et le riz servi aux participants. Ce riz provenait des « rizières du sanctuaire » shinden et il était fourni par les districts du sanctuaire au titre de taxe foncière.

Les « Règlements de l'ère Engi », qui sont postérieurs de plus d'un siècle aux deux rapports du sanctuaire d'Ise, précisent (ES, p. 195) que chacune des vingt jarres de saké d'offrande contenait 3 to (21 litres) et que le saké était prélevé sur les ressources du sanctuaire shinzei, constituées par toutes les recettes fiscales. Sur ces vingt jarres, quinze étaient livrées à Ise par les trois districts du sanctuaire et les cinq autres par les provinces d'Iga, d'Owari, de Sanuki et de Tôtômi, ce qui prouve que les ressources $\mathrm{du}$ sanctuaire ne provenaient pas seulement de la province d'Ise. Les « Règlements de l'ère Engi », nous informent en outre sur les denrées fraîches utilisées à l'occasion des rites (ES, p. 67). Pour le rite des « Travaux agricoles » célébré à la cour impériale et dans trois cent quatre sanctuaires du pays, les offrandes de denrées fraîches comprenaient de la bonite, des ormeaux, de la chair séchée, des algues et du sel qui étaient présentés dans des « emballages de nattes de paille » hagomo tsutsumi de cinq pieds (1,5 mètre). On peut donc estimer que les denrées fraîches présentées en offrande au sanctuaire d'Ise comprenaient le même type de produits, à savoir des produits de la mer, en particulier des fruits de mer, des algues et du sel.

La deuxième tâche principale du sanctuaire était l'offrande quotidienne mike, qui avait lieu matin et soir, dans les deux sanctuaires intérieur et extérieur. Pour cette offrande, le sanctuaire devait disposer de deux pièces de soie, huit pièces de toile de chanvre, trois sièges mushiro de chanvre et trois stores de chanvre (Tg, p. 201) qui étaient renouvelés tous les jours et jamais réutilisés. Ces chiffres sont à multiplier par 365 pour l'année et il fallait aussi des milliers de coupelles en terre cuite hajiki qui étaient, elles aussi, utilisées une seule fois, puis jetées. Les offrandes quotidiennes se composaient de quatre bols d'eau, vingt-huit portions de riz cuit, quatre coupelles de sel et de denrées fraîches (Tg, p. 201), comprenant en principe du poisson, des légumes et des algues. Les offrandes étaient préparées tous les jours, à tour de rôle, 
par des desservants désignés qui passaient la nuit dans la « résidence purifiée » imitachi ou saikan. À l'aurore ils se rendaient dans la cuisine au feu purifié et présentaient aux dieux un repas sacré mike dans le " pavillon des repas sacrés » mike-den (Yano, p. 118-119). Le riz des offrandes quotidiennes provenait des « rizières du sanctuaire ». Mais le riz servi, matin et soir, lors des trois grands rites était cultivé dans les « rizières sacrées » mita du sanctuaire et conservé dans le grenier mikura situé dans l'enceinte du sanctuaire (Kjg, p. 143, 152).

Cette année, nous n'avons pas pu étudier les bâtiments des deux sanctuaires intérieur et extérieur et les travaux d'entretien auxquels ils donnaient lieu. Le pavillon principal où la déesse Amaterasu est censée résider est reconstruit, en principe, tous les vingt ans, ce qui occasionnait des dépenses importantes. Nous avons précédemment consacré des séances à ce sujet, dans le cadre de notre conférence sur " Les outils dans la société de Heian » (voir Annuaire de l'EPHE 2004-2005). La « résidence purifiée » comprenait un pavillon purifié imidono de deux toises (6 mètres) sur une toise ( 3 mètres) et huit pieds (2,4 mètres) de hauteur, des cuisines pour étuver le riz kashigiya, un atelier du fourneau à feu sacré imibi kashigiya pour le riz d'offrande et une cuisine pour préparer le riz ôiya destiné aux repas du personnel, un grenier, un gardemanger kuriya et les étables umaya du cheval sacré. Le tout était entouré d'une palissade d'une longueur de 150 mètres (Kjg, p. 23).

Le personnel du sanctuaire d'Ise était composé de fonctionnaires administratifs, du personnel affecté au culte, d'employés chargés des tâches techniques et d'une main d'oeuvre subalterne. L'administrateur gûji était un fonctionnaire détaché par le ministère des Affaires des dieux de la cour de Heian. Pour les deux sanctuaires intérieur et extérieur, le nombre de personnes affectées au culte a varié avec le temps, mais il se composait en moyenne d'une trentaine à une quarantaine de personnes pour le sanctuaire intérieur (ES, p. 185, Kjg, p. 98) et de quatre-vingt six personnes pour l'ensemble du sanctuaire en 927 (ES, p. 255). Les rites annuels et quotidiens étaient dirigés respectivement par un desservant negi, appellation qui signifie « prieur », une fonction héréditaire remplie par les familles Arakida et Watarai respectivement pour les sanctuaires intérieur et extérieur. Les negi qui étaient des fonctionnaires (Torao, p. 804), habitaient dans la « résidence purifiée » et ils étaient, en permanence, soumis à l'interdit.

Le desservant était assisté par trois ou quatre «grands assistants de l'intérieur» ôchibito (ouchindo) dont le plus important habitait dans la « résidence purifiée ». Ces personnes étaient choisies dans les familles Isobe, Arakida et Kannushi et ont été élevées au statut de fonctionnaire au $\mathrm{IX}^{\mathrm{e}}$ siècle (Torao, p. 184, 805). Les neuf à treize jeunes filles et jeunes garçons en service au sanctuaire monoimi étaient accompagnés, à l'origine, par leur pères monoimi no chichi, et plus tard, par des hommes qui se tenaient à leurs côtés. Ces enfants étaient issus de familles de notables locaux des districts du sanctuaire Ise et ils accomplissaient des tâches pratiques et rituelles au service des dieux et plus particulièrement Amaterasu. Ils fabriquaient la vaisselle d'offrande en poterie et le saké sacré, grillaient le sel, ou encore étaient affectés comme gardes aux portes des lieux où résidaient les divinités. Soumis à l'interdit et maniant les objets destinés aux dieux, ils habitaient à la « résidence purifiée » (Torao, p. 806, 807). Il y avait aussi neuf à treize " petits assistants de l'intérieur » kouchibito qui étaient artisans et techniciens, potiers, forgerons, ébénistes et éleveurs de chevaux. 
Les frais de personnel du sanctuaire d'Ise sont difficiles à évaluer. Sur les quatrevingt six personnes actives dans l'administration ou le culte des sanctuaires intérieur et extérieur, seuls deux administrateurs, deux adjoints, un directeur des célébrations saishu, deux desservants, et plusieurs ôchibito avaient un traitement de fonctionnaire au $\mathrm{X}^{\mathrm{e}}$ siècle (ES, p. 247, 249). Ces personnes percevaient des allocations semestrielles constituées de taffetas de soie (ES, p. 341), ou de pongé et de bourre de soie, de toile de chanvre, et de fer sous forme de lingots ou de houes, comme les fonctionnaires de même grade à la cour de Heian. Les titulaires des quatrième ou cinquième rangs avaient droit, en outre, à une dizaine ou une vingtaine d'hectares de « rizières de rang » iden, et ils avaient à leur service plusieurs dizaines de domestiques shijin qui venaient des districts du sanctuaire. Lors de leur prise de fonction, le directeur des célébrations et les deux administrateurs étaient respectivement gratifiés de dix mille gerbes et de mille gerbes de riz. Tous, jusqu' au grade de monoimi, recevaient en outre du riz au titre de per diem dont la quantité était supérieure à celle des repas quotidiens (ES, p. 247, 249).

Mis à part le personnel réservé au culte, il y avait aussi un devin, dix-huit danseurs, sept musiciens et quinze employés de bureau qui, tous, venaient des trois districts du sanctuaire et « des foyers concédés au sanctuaire dans six autres lieux » (ES, p. 245251). On peut supposer que ces personnes, comme tout le personnel subalterne, étaient logées et nourries par le sanctuaire.

Le personnel subalterne du sanctuaire se composait de quatre-vingt six corvéables, hommes à tout faire shichô, et d'un nombre inconnu de corvéables artisans zôyô ou zôeki, venant des foyers des trois districts du sanctuaire, des préposés à diverses tâches zôshiki et des techniciens du rite kanbe dont on ignore aussi le nombre, ainsi que de cinquante tisserands kanhatori et kan'omi et de dix-huit éleveurs de chevaux auxiliaires umakaichô (ES, p. 243, 245, 253, 255). Le personnel d'Ise comprenait donc deux cent soixante-six personnes avant 927, date de la publication des « Règlements de l'ère Engi » et une quantité non précisée de personnel subalterne que l'on peut évaluer à trois cents personnes, ce qui donne un total de cinq cents à six cents personnes. Cette année a été consacrée à deux volets de la comptabilité du sanctuaire, à savoir les célébrations et les offrandes rituelles, d'une part, et le personnel, d'autre part.

Pour certaines offrandes rituelles, le sanctuaire d'Ise percevait le produit de 36 hectares de « rizières du sanctuaire » shinden, selon Torao (p. 207, 255, 832) et Kamata (p. 274-275), mais on peut se demander s'il n'y a pas, dans ce cas, confusion avec les « rizières sacrées » mita, gérées directement par le personnel du sanctuaire. Les « rizières du sanctuaire » comportaient, quant à eux, des centaines d'hectares qui étaient dispersés dans plusieurs « districts attachés au sanctuaire ». Quatre districts, situés aux alentours du sanctuaire, lui versaient la totalité des prestations fiscales, levées sur leurs "foyers attachés au sanctuaire», à savoir les taxes en nature, les taxes remplaçant la corvée, la taxe foncière et les corvées. Plusieurs autres « districts du sanctuaire », situés à Ise et dans six autres provinces payaient au sanctuaire la taxe foncière de leurs « foyers attachés au sanctuaire ». Ces versements constituaient les ressources principales du sanctuaire shinzei d'Ise (Kamata, p. 272, 276-298, Hérail, p. 24-29, Torao, p. 173, 257-257, 793-795, 829, 832). L'économie du sanctuaire reposait donc essentiellement sur les « foyers du sanctuaire », dont cent quarante-quatre 
habitaient dans les quatre district près du sanctuaire et trois cent dix-sept autres foyers à Ise et dans les autres provinces, selon les chiffres des «Règlements de l'ère Engi » (ES, p. 257). Mais ces chiffres diffèrent en fonction des décomptes et ils ont évolué dans le temps (Kamata, p. 275, 286). Le sanctuaire recevait des denrées fraîches, des textiles et de l'artisanat et levait les corvées selon ses besoins, au cas par cas, tandis que la gestion des ressources en riz se faisait à l'année.

La cour impériale, le gouvernement de la province d'Ise et l'administration du sanctuaire étaient tous trois impliqués dans la gestion des ressources du sanctuaire d'Ise. Celui-ci tenait la comptabilité des dépenses en riz, mais il était en concurrence avec le gouverneur de la province d'Ise pour le contrôle des prélèvements dans les foyers attachés au sanctuaire dans cette province. La comptabilité des recettes et des livraisons d'Ise était obligatoirement soumise à l'approbation du ministère des Affaires des dieux de la cour impériale. En 815, les dépenses du sanctuaire d'Ise s'élevaient à 41190 gerbes de riz, les recettes à 36585 gerbes, soit un déficit de 4650 gerbes (Ruiju sandai kyaku, livre 1, Hérail, p. 24-30). Ces chiffres confirment nos estimations concernant les besoins en riz du sanctuaire. Nous avons, plus haut, évalué les besoins pour les trois grands rites de l'année à 12 tonnes de riz. Avec une moyenne de 834 grammes de riz décortiqué par gerbe, cela donne 14388 gerbes. Cette dépense représentait un tiers des dépenses totales consacrées au culte pour l'année 815. L'étude des deux «Rapports sur le protocole du Sanctuaire impérial d'Ise » de 804 nous a permis de mettre en lumière le fonctionnement de l'économie du sanctuaire, mais nous n'avons encore exploité qu'une infime partie des données qu'ils contiennent sur la culture matérielle et la vie pratique du premier sanctuaire du Japon.

\section{Bibliographie}

Bock (Felicia), Engi-shiki Procedures of the Engi Era, Books I-V, Sophia University Press, 1970. Emura Hiroyuki, « Jingû gishikichô ni mieru nie ni tsuite », Naoki Kôjirô sensei koki kinenkai (éd.), Kodaishi ronshû,ge, Tokyo, Hanawa Shobô, 1989.

Hérail (Francine), Recueil de décrets des trois ères méthodiquement classés, livres 1 à 7 , Genève, Librairie Droz, 2011 (Hautes Études orientales, 50).

Jingû shuchô (éd.), Jingûshi nenpyô, Tokyo, Ebisu Kôshô Shuppan, 2005.

Kumata Ryônosuke, « Ritsuryô-ka Ise jingû no keizaiteki kiban to sono tokushitsu », Seki Akira kyôju kanreki kinenkai (éd.), Nihon kodaishi kenkŷu, Tokyo, Yoshikawa Kôbunkan, 1980.

Ôzeki Kunio, « Kodai Ise jingû no zaisei kôzô », Kokushigaku, 128 (février 1986).

Tokyo kokuritsu habubutsukan (éd.), Ise jingû to kamigami no bijutsu, Tokyo, Kasumi Kaikan, 2009.

Torao Toshiya (éd.), Engishiki, vol. 1, Tokyo, Shûeisha, 2005.

Yano Kenichi, Ise jingû no ishokuju, Tokyo, Tôkyô Shoseki, 1992.

\section{II. «Chronique des relations avec les pays voisins » (Zenrin kokuhôki, 1470), texte en kanbun}

La «Chronique des relations avec les pays voisins » (1470) est une compilation du moine Zen Zuikei Shûhô (1391-1473) qui retrace l'histoire des relations du Japon avec la Chine et la Corée dans l'ordre chronologique avec des commentaires de l'auteur, des extraits de sources japonaises et chinoises, et plusieurs copies de lettres qui 
ne figurent dans aucune autre source historique. Nous avons examiné précédemment la correspondance diplomatique du shogun Ashikaga Yoshimitsu (1358-1408), et celle qui concerne la politique extérieure du shôgun suivant Ashikaga Yoshimochi (13861428) (voir les Annuaires de l'EPHE des années 2004-2005 et 2008-2009). Cette année nous avons continué la lecture de ce texte avec les mêmes éditions, à savoir celle de Ishii Masatoshi (1995), le manuscrit inédit du Zenrin kokuhôki de la bibliothèque de l'université de Cambridge (Catalogue des ouvrages japonais, « Gaikoku kankei » $n^{\circ} 1384$, collection George Aston, 1911), manuscrit daté le plus ancien si l'on en croit le post-scriptum de l'année 1568, et l'édition xylographique de 1657 (Zenrin kokuhôki, Tokyo, Kokusho Kankôkai, 1975).

Nous avons lu plusieurs pièces consignées dans le Zenrin kokuhôki, dont les cinq premières documentent les relations du shogun Ashikaga Yoshimochi avec le roi de Corée, Sejong (r. 1418-1450). Après avoir interrompu les relations avec la Chine en 1419 , le shogun s'est tourné vers la Corée dans un but bien précis. Dans sa lettre datée de 1422, cinquième mois, le shogun demande qu'on lui fasse parvenir l'édition xylographique coréenne du Canon bouddhique en sept mille rouleaux. La lettre suivante du shogun au roi, datée de 1423, septième mois, le remercie pour le don du Canon bouddhique et demande, cette fois, les planches gravées de la compilation bouddhique. Le shogun renouvelle sa demande dans une lettre, l'année suivante, à la huitième lune. En 1425 , à la cinquième lune, le roi coréen répète qu'il ne se séparera pas du seul exemplaire existant des planches xylographiques. En 1428, au troisième mois, une lettre est adressée au roi coréen au nom du shogun Yoshimochi, pour demander une nouvelle copie imprimée du Canon bouddhique, cette fois à l'intention du temple Tôfukuji de Hakone, demande qui aurait été faite par l'intermédiaire du représentant à Kamakura du shogun de Kyoto. Cette lettre est un faux dont il n'y a pas de trace dans les chroniques coréennes et qui nous a donné l'occasion d'examiner un écrit de style médiocre. Durant son règne de 1409 à 1428, le shogun Yoshimochi a envoyé six missions en Corée et a reçu cinq délégations de ce pays. Il a demandé à plusieurs reprises le Canon bouddhique, une compilation de mille cinq cent seize textes bouddhiques, pour la plupart des traductions chinoises de textes indiens écrits en sanscrit. Cette compilation a été imprimée en Chine au $\mathrm{X}^{\mathrm{e}}$ siècle, puis en Corée au $\mathrm{XI}^{\mathrm{e}}$ siècle. Après la disparition de ces éditions lors des invasions mongoles, la Corée a publié une nouvelle version à partir de quatre-vingt-onze mille deux cent cinquante-huit planches en six mille huit cent quinze rouleaux. On comprend que les demandes insistantes du shogun japonais aient été peu appréciées par le roi de Corée. Entre 1388 et le milieu du XVI ${ }^{\mathrm{e}}$ siècle, une centaine de requêtes pour obtenir ce précieux recueil ont été adressées à la cour coréenne par les shogun, les gouverneurs militaires des provinces et les élites du sud du Japon et de Tsushima. Le Canon bouddhique était très recherché, non seulement pour sa valeur religieuse, mais aussi en tant que symbole de prestige et de pouvoir. Peu de chefs militaires du Japon ont réussi à en obtenir une copie du roi de Corée.

Après la mort de Yoshimochi en 1428, son frère, le shogun Yoshinori (1494-1441) a envoyé une ambassade à la cour chinoise de la dynastie des Ming, en 1432. Sa lettre datée de 1429, troisième lune, adressée à l'empereur Xuanzong (r. 1425-1435), propose de reprendre les missions japonaises portant le tribut en Chine, après un répit de dix ans. Cette lettre a été présentée à la cour chinoise par un envoyé du shogun, 
moine de l'école Zen, qui était accompagné d'une importante délégation commerciale de cinq navires de marchands. Il va sans dire que le shogun agissait pour les intérêts économiques du gouvernement militaire de Kyoto. L'empereur de Chine répondit par une missive datée de 1433, sixième lune, qu'il a fait porter par un délégué chinois qui a accompagné les envoyés japonais à leur retour de Beijing à Kyoto. Dans cet écrit il exalte la puissance de l'Empire du milieu, rappelle les règles du protocole du tribut et loue les mérites du shogun Yoshimitsu, père de Yoshinori, qui avait envoyé de nombreuses missions tributaires en Chine. Cet écrit nous a servi de modèle pour mettre en évidence une terminologie diplomatique chinoise fortement imprégnée de morale confucéenne à commencer par les notions de rites et de devoir, l'influence bénéfique de la voie royale, la grâce du ciel, la clémence et la bienveillance du souverain, ainsi que la loyauté, la piété filiale et la sincérité des vassaux. En même temps, en 1433 , le souverain chinois a remit un certificat à l'envoyé japonais - un moine né en Chine - qui lui conférait un titre monastique chinois et il l'a encouragé à en faire usage pour sa carrière dans les monastères de Kyoto, s'arrogeant ainsi le droit d'intervenir dans les affaires religieuses du shogun. Celui-ci profita de la réponse positive du souverain chinois pour déléguer aussitôt une nouvelle mission en Chine accompagnée de six bateaux de commerce. Cette mission présenta au souverain des Ming une lettre datée de 1434, huitième lune, dans laquelle le shogun confirmait son allégeance comme " serviteur à l'égard du Grand ». La délégation japonaise revint au Japon avec une lettre de l'empereur Yingzong (r. 1435-1449), datée de 1436, deuxième mois, qui reprenait le discours protocolaire du suzerain adressé à son vassal. Il n'y eut que ces deux échanges de missions avec la Chine durant le règne du shogun Yoshinori qui échangea par ailleurs seulement deux missions avec la Corée. La lecture de ces quelque dix courriers diplomatiques a fait apparaître le traitement différent réservé par les deux shogun à leurs partenaires. Les lettres adressées à la Corée sont écrites dans un style soutenu mais leur contenu est strictement limité aux affaires, alors que celles envoyées en Chine sont remplies d'éloges du souverain chinois. Le shogun confiait la rédaction de ses lettres au souverain chinois à des moines, poètes et écrivains éminents de son temps. Il n'attendait pas la même chose de la Corée que de la Chine. De la première, il souhaitait obtenir le Canon bouddhique, alors qu'il envisageait de faire du commerce de grande envergure avec la seconde. Ainsi les courriers que nous avons étudiés cette années, nous ont permis d'analyser le vocabulaire diplomatique utilisé par les souverains des trois pays de l'Asie orientale.

\section{Bibliographie}

Ishii Masatoshi (éd.), «Zenrin kokuhôki », Tanaka Takeo (éd.), Zenrin kokuhôki, Shintei zoku zenrin kokuhôki, Tokyo, Shûeisha, 1995.

Robinson (Kenneth), « Centering the King of Choson: Aspects of Korean Maritime Diplomacy, 1392-1592», The Journal of Asian Studies, 59, 1 (février 2000).

Verschuer (Charlotte von), Le commerce extérieur du Japon des origines au XVIe siècle, Paris, Maisonneuve \& Larose, 1988.

Verschuer (Charlotte von), «Ashikaga Yoshimitsu's Foreign Policy 1398 to 1408 A.D. A Translation from Zenrin Kokuhôki, the Cambridge Manuscript », Monumenta Nipponica, 62, 3 (automne 2007). 